\title{
DIABETES MELITTUS: BUSCA ATIVA EM PORTADORES DE OBESIDADE*
}

\author{
Elaine Braga Faustino', Elisângela Braga de Azevedo², Priscilla Maria de Castro Silva ${ }^{3}$, Sandra dos Santos Sales ${ }^{4}$, \\ Walnísia Polyanna de Sousa Barros ${ }^{5}$
}

\begin{abstract}
RESUMO: O Diabetes Mellitus configura-se um dos maiores problemas de saúde pública, levando seus portadores a complicações de saúde. Esta pesquisa teve por objetivo investigar casos suspeitos de Diabetes Mellitus por meio de busca ativa entre portadores de obesidade de uma Unidade Básica de Saúde da Família do Município de Campina Grande, Estado da Paraíba. Trata-se de um estudo descritivo, exploratório e quantitativo, cuja amostra foi constituída por 103 usuários, 21,4\% homens e 78,6\% mulheres. Os resultados relativos ao Índice de Massa Córporea evidenciaram que 5,8\% dos sujeitos apresentavam risco aumentado para desenvolver diabetes. A ação investigatória chama a atenção para a problemática, sugerindo a necessidade de atividades educativas e da prática cotidiana de busca ativa para a detecção precoce dos casos de risco para o Diabetes Mellitus.
\end{abstract}

PALAVRAS-CHAVE: Programa saúde da família; Diabetes mellitus; Obesidade.

\section{DIABETES MELLITUS: AN ACTIVE SEARCH AMONG THE OBESE}

\begin{abstract}
Diabetes Mellitus is one of the biggest problems for public health, as it leads to further health problems. This research aimed to investigate suspected cases of Diabetes Mellitus by means of an active search among obese patients in a Basic Family Health Unit of Campina Grande county in the state of Paraíba. It is a descriptive, exploratory and quantitative study, whose sample was composed of 103 subjects, of whom $21.4 \%$ were men and $78.6 \%$ women. Related to the Body Mass Index the results indicated that $5.8 \%$ of the subjects presented an increased risk of developing diabetes. The investigative action calls attention to the problem, suggesting the need for educational activities and for the routine practice of active search for the early detection of risk cases for Diabetes Mellitus.
\end{abstract}

KEYWORDS: Family health programs; Diabetes mellitus; Obesity.

\section{DIABETES MELITTUS: BÚSQUEDA ACTIVA EN PORTADORES DE OBESIDAD}

RESUMEN: El Diabetes Mellitus constituye uno de los mayores problemas de salud pública, traendo complicaciones de salud a sus portadores. Esta investigación tuvo por objetivo investigar casos sospechosos de Diabetes Mellitus por medio de búsqueda activa entre portadores de obesidad de una Unidad Básica de Salud de la Familia del municipio de Campina Grande, estado de Paraíba. Es un estudio descriptivo, exploratorio y cuantitativo, cuya muestra fue compuesta de 103 usuarios, 21,4\% hombres y 78,6\% mujeres. Los resultados acerca del Índice de Masa Corpórea evidenciaron que $5,8 \%$ de los sujetos presentavan gran riesgo de desarrollar diabetes. La acción investigativa llama atención para la problemática, sugiriendo la necesidad de actividades educativas y de práctica cotidiana de búsqueda activa para la detección precoz de los casos de riesgo del Diabetes Mellitus.

PALABRAS CLAVE: Programa salud de la familia; Diabetes mellitus; Obesidad.

\footnotetext{
* Este artigo é um recorte do trabalho de conclusão do Curso de Graduação em Enfermagem apresentado à UNESC, em 2009. ${ }^{1}$ Enfermeira.

${ }^{2}$ Enfermeira Sanitarista. Professora do Departamento de Enfermagem da Faculdade Maurício de Nassau. Mestranda em Enfermagem na Universidade Federal da Paraíba-UFPB. Membro do Grupo de Estudos e Pesquisas em Saúde Mental Comunitária.

${ }^{3}$ Enfermeira. Especialista em Saúde Mental. Professora do Departamento de Enfermagem da Faculdade de Ciências Médicas de Campina Grande-FCM.

${ }^{4}$ Enfermeira. Especialista em Saúde Pública. Professora do Departamento de Enfermagem da Universidade Estadual da Paraíba.

${ }^{5}$ Enfermeira. Especialista em Saúde Pública e em Saúde do Trabalhador. Preceptora de estágio da FCM.
}

Autor correspondente:

Elisângela Braga de Azevedo

Faculdade Maurício de Nassau

R. Pedro Soares da Silva, 55 - 58105-411 - Catolé-PB-Brasil

Recebido: 08/10/10

E-mail: elisaaz@terra.com.br Aprovado: 09/02/11 


\section{INTRODUÇÃO}

O Diabetes Mellitus (DM) configura-se como uma epidemia mundial, traduzindo-se em grande desafio para os sistemas de saúde de todo mundo. Sua ocorrência na população brasileira mostra-se de forma cada vez mais crescente, estimando-se que até 2025, o número de diabéticos chegue a 11 milhões de portadores no país ${ }^{(1)}$. No final da década de 1980, segundo estudos epidemiológicos, esse agravo à saúde ocorria em cerca de $8 \%$ da população de 30 a 69 anos de idade, residentes em áreas metropolitanas brasileiras, variando de 3 a $17 \%$ entre as faixas de 30 a 39 e de 60 a 69 anos, respectivamente. A prevalência da tolerância à glicose diminuída era igualmente de $8 \%$, variando de 6 a $11 \%$ entre as mesmas faixas etárias ${ }^{(1)}$.

Por sua vez, o envelhecimento crescente da população, a urbanização acelerada e a adoção de estilos e hábitos alimentares pouco saudáveis vêm aumentando esta estatística e, a cada dia, as pessoas apresentam modos de vida sedentária, alimentação inadequada e, por vezes, obesidade mórbida, sendo este um dos fatores preocupantes para a incidência e a prevalência do diabetes em todo o mundo (2).

Um estudo epidemiológico mostrou a incidência progressiva de doenças crônicas como Hipertensão Arterial, Diabetes Mellitus tipo 2, Dislipidemia e Doenças Coronarianas com níveis elevados de Índice de Massa Corpórea (IMC). Nas práticas assistenciais esses níveis também se mostram alterados, sendo detectados pelos profissionais de saúde por meio da medida da circunferência da cintura e pela relação cintura/quadril ${ }^{(3)}$.

A obesidade tem adquirido grande proporção e, consequentemente, causado mais problemas para a saúde da população, levando à descompensações diabéticas e impactando a qualidade de vida dos indivíduos. A obesidade caracteriza-se pelo excesso de tecido adiposo, provocando aumento de peso e refletindo no número aproximado de 300 milhões de pessoas obesas em todo o mundo ${ }^{(4)}$.

Um dispositivo de atenção à saúde que vem melhorando a qualidade da assistência oferecida à população é a Estratégia Saúde da Família (ESF); prioriza ações de promoção, proteção e recuperação da saúde dos indivíduos e da família de forma integral e contínua. A partir de janeiro de 1994 começaram a ser formadas as primeiras Equipes de Saúde da Família, na perspectiva de construção de um novo modelo assistencial, tendo por princípios a descentralização, municipalização, integralidade e qualidade das ações assistenciais ${ }^{(5)}$.

A ESF é um projeto dinamizador do Sistema Único de Saúde (SUS), e está condicionada pela evolução histórica e pela organização do sistema de saúde no Brasil. A grande velocidade de expansão da Saúde da Família comprova a adesão de gestores estaduais e municipais aos seus princípios, apresentando um crescimento expressivo nos últimos anos. A consolidação dessa estratégia precisa, entretanto, ser sustentada por um processo que permita a real substituição da rede básica de serviços tradicionais no âmbito dos municípios e pela capacidade de produção de resultados positivos nos indicadores de saúde e de qualidade de vida da população assistida.

Uma das estratégias utilizadas na atualidade para o rastreamento do DM nos serviços de saúde se dá a partir da busca ativa dos sinais e sintomas e das condições de risco. Por sua vez, a glicemia capilar pode ser utilizada para o rastreamento da doença, devendo-se confirmar o diagnóstico com glicemia plasmática ${ }^{(6)}$.

O DM apresenta-se com incidência e prevalência crescentes. Neste contexto, justificamos a relevância da pesquisa no âmbito da ESF por priorizar a prevenção de doenças e a promoção da saúde. No entanto, poucos investimentos são feitos por parte dos profissionais e gestores da saúde no que concerne a trabalhos de sensibilização quanto aos reais riscos a que portadores de obesidade estão expostos. Ressalta-se à necessidade de aconselhamentos quanto aos hábitos alimentares como forma de prevenir o DM.

Este trabalho buscou promover o rastreamento do DM em obesos, pois sabe-se que a obesidade é um dos principais fatores que levam ao desenvolvimento dessa patologia. Corroborando com essa ideia, pesquisadores referem que o peso corpóreo pode ser considerado um fator de risco que resulte no DM. De acordo com a Comissão Nacional de Diabetes dos Estados Unidos, o risco dessa doença desenvolver é de duas vezes maior em obesos leves, cinco vezes em obesos moderados e de dez vezes em obesos severos ${ }^{(7)}$.

A estratégia da busca ativa do DM e outras patologias, junto a grupos de risco, constitui excelente oportunidade para o diagnóstico precoce e consequente prevenção de complicações.

Sendo assim, este estudo teve como objetivo identificar casos suspeitos de DM através de busca ativa em portadores de obesidade cadastrados na Unidade Básica de Saúde da Família Tambor I do Município de Campina Grande, Estado da Paraíba. Especificamente, buscou-se classificar os portadores de obesidade por 
meio do cálculo de Índice de Massa Corpórea (IMC), traçar o perfil epidemiológico dos portadores de obesidade, mensurar a circunferência abdominal dos pesquisados, e identificar a ocorrência de Hipertensão Arterial Sistêmica (HAS) e sua associação com o DM.

\section{METODOLOGIA}

Trata-se de um estudo descritivo, exploratório, investigativo de abordagem quantitativa. Na pesquisa quantitativa, o pesquisador observa, registra, analisa e correlaciona fatos e fenômenos (variáveis) sem manipulá-los. Sua preocupação é descobrir a freqüência em que o fenômeno ocorre, sua relação e conexão com outros fenômenos, sua natureza e características ${ }^{(8)}$.

O universo da pesquisa foi constituído de todos os usuários da Unidade Básica de Saúde da Família (UBSF) do Tambor I do Município de Campina Grande-PB, porém, a amostra foi constituída de 103 usuários portadores de obesidade que atenderam aos seguintes critérios de inclusão: ser portador de obesidade com IMC > 30; ter entre 35 a 60 anos de idade; ser cadastrado na UBSF; ter disponibilidade em participar da pesquisa; e assinar o Termo de Consentimento Livre e Esclarecido.

$\mathrm{O}$ instrumento utilizado para a coleta dos dados foi um questionário com dez questões fechadas e preenchido durante a consulta no domicílio do usuário pesquisado. Foram utilizados os seguintes instrumentos para subsidiar a assistência e a coleta dos dados: balança digital; fita antropométrica; glicosímetro; fita de glicemia capilar, tensiômetro e esfignomanômetro; luvas; lanceta; algodão e caixa para perfurocortantes.

A coleta de dados ocorreu nos meses de setembro a outubro de 2009 e a aplicação do questionário ocorreu por meio da busca ativa nos domicílios que foram selecionados, de acordo com os critérios de inclusão descritos acima. Desse modo, foi realizada a glicemia capilar, conferidos peso, altura e circunferência abdominal, aferida aa pressão arterial e, posteriormente, aqueles que tiveram alterações comprovadas foram encaminhados à UBSF para exames complementares. As informações foram coletadas e registradas no questionário.

Os dados obtidos foram tabulados e analisados quantitativamente pela frequência absoluta e percentual e apresentados sob a forma de porcentagens. O programa utilizado foi o Microsoft Word versão 2003 e o Excel 2003.

A pesquisa foi submetida ao Conselho de Ética da Universidade Estadual da Paraíba com aprovação sob protocolo n. 03720133000-09. Foram atendidas as exigências do código de ética em pesquisas envolvendo seres humanos ${ }^{(9)}$.

\section{RESULTADOS}

A população inserida no estudo totalizou 103 usuários, sendo $81(78,6 \%)$ do sexo feminino e 22 $(21,4 \%)$ do sexo masculino.

Com relação à distribuição percentual do grau de obesidade e sobrepeso encontrados no sexo feminino, observa-se uma incidência de obesidade grau I de $45,6 \%$, obesidade grau II com $21,4 \%$, obesidade grau III de 5,8\%. Considerando que o sobrepeso também representa um fator de risco para o desenvolvimento do DM, encontramos um percentil de $5,8 \%$ de pacientes portadores dessa patologia, o que consideramos um dado importante desta pesquisa.

$\mathrm{Na}$ distribuição percentual do grau de obesidade e sobrepeso encontrados no sexo masculino observase um percentil de $15,5 \%$ de obesidade grau I, 3,9\% de obesidade grau II e 1,0\% apresentaram obesidade grau III, como também sobrepeso.

Os resultados dispostos em percentuais destacam que os portadores de obesidade da UBSF Tambor I encontram-se com a circunferência abdominal com risco muito aumentado, perfazendo um total de $94,2 \%$ da amostra e um percentil de 5,8\% da população com risco aumentado para desenvolver DM. Percebemos que essa medida é um fator de risco para o desenvolvimento da doença, portanto, a equipe precisa alerta-se quanto a esse dado.

Foi identificado que existe uma relação da glicemia capilar pós-prandial entre o sexo feminino e o sexo masculino, com resultados de glicemia capilar $<140 \mathrm{mg} / \mathrm{dl}$ encontrados em 50,5\% das mulheres e em $14,6 \%$ dos homens. Sobretudo, na população pesquisada, houve uma apresentação de glicemia $>140 \mathrm{mg} /$ dl, distribuídas da seguinte forma: mulheres- $28,2 \%$ e homens- $6,8 \%$.

$\mathrm{Na}$ distribuição percentual da pressão arterial sistêmica da população do sexo feminino, destacou-se um índice de $38,9 \%$ de normotensos; $28 \%$ de portadores de hipertensão leve; $8,8 \%$ de hipertensão grave e $2,9 \%$ dos pesquisados tinham hipertensão moderada. Na distribuição percentual da pressão arterial sistêmica na população do sexo masculino, destacou-se um índice de $8,7 \%$ de normotensão; $3,9 \%$ eram portadores de hipertensão leve; $5,9 \%$ de hipertensão moderada e 


\section{2,9\% com hipertensão grave.}

No tocante ao percentual do histórico familiar de morbidades referidas pela população pesquisada, foram encontradas $81,6 \%$ de casos familiares de hipertensão arterial sistêmica; $73,8 \%$ de obesidade, $54,5 \%$ de DM; $38,9 \%$ de cardiopatias e, $2 \%$ de casos de dislipidemias. Contudo, quanto aos antecedentes pessoais relatados pela amostra, verificou-se que $82,5 \%$ referiram obesidade, $59,4 \%$ eram hipertensos e $3 \%$ disseram já ter apresentando, em algum momento de suas vidas, hiperglicemia. Este foi mais um motivo para se realizar rastreamento nestes pacientes, já que pelo grau de exposição tanto familiar quanto pessoal, estes usuários apresentam risco potencial para desenvolver a patologia estudada.

Com relação à distribuição percentual do IMC e hiperglicemia na população do sexo feminino, com faixa etária de 51 a 60 anos, encontramos 20,4\% com prevalência de obesidade e hiperglicemia e nas faixas etárias de 35 a 50 anos do mesmo sexo, encontramos $4,8 \%$. Na população do sexo masculino, encontramos nas faixas etárias de 51 a 60 anos $3,9 \%$ com prevalência de obesidade e hiperglicemia.

\section{DISCUSSÃO}

O DM tipo 2 (DMT2) configura-se por uma deficiência do pâncreas em produzir insulina; cerca de $80 \%$ dos usuários podem receber assistência na atenção básica, quando diagnosticado, porém, um fator agravante é que $50 \%$ da população não sabe ser portadora da doença, permanecendo sem diagnóstico até que se manifestem os primeiros sinais de complicação. Dessa forma, o rastreamento é indicado em indivíduos assintomáticos que apresentem maior risco da doença, favorecendo, assim, um diagnóstico precoce ${ }^{(10)}$.

A importância da busca ativa do DM em pacientes com obesidade se dá pela possibilidade de identificar casos novos da patologia. Previamente, estes casos podem ser diagnosticados com a realização da glicemia capilar, devendo ser confirmados com glicemia plasmática. Mas, o diagnóstico também pode ser complementado por um levantamento de informações a respeito da idade, ocupação, condições mórbidas, uso de medicação e, entre outros fatores, a existência de familiares com diabetes, especificando o grau de parentesco e os possíveis riscos a que estes estão $\operatorname{expostos}^{(6)}$.

As prevalências de excesso de sobrepeso e obesidade na população adulta brasileira revelam que esses agravos alcançam grande expressão em todas as regiões do país, no meio urbano e rural, e em todas as classes sociais. A obesidade se caracteriza por IMC igual ou superior a $30 \mathrm{~kg} / \mathrm{m} 2$, afeta $8,9 \%$ dos homens adultos e 13,1\% das mulheres adultas do país. Sendo assim, percebe-se que as pesquisas demonstram uma prevalência de obesidade bem maior em mulheres que, consequentemente, apresentam maior risco de desenvolver DMT2 ${ }^{(11)}$.

Os dados dessa pesquisa não diferem muito de outros estudos que apontam a população masculina adulta com prevalência de obesidade em $11,2 \%$, sendo esse desvio nutricional associado aos novos estilos de vida, hábitos alimentares, sedentarismo, como também à hipertensão arterial e às intercorrências cardiovasculares/cardiocirculatórias ${ }^{(12)}$.

Entretanto, este estudo revelou que a população feminina, de forma especial, vem sendo mais atingida com a obesidade, podendo interferir na autoestima, na saúde e, consequentemente, na qualidade de vida ${ }^{(13)}$.

Sobretudo, além do excesso de gordura corpórea, deve-se considerar ainda sua distribuição espacial, uma vez que o excesso de gordura localizada na região abdominal é um fator de risco maior de morbidade que o excesso de gordura corpórea em si. Isso justifica que, para identificar os fatores de risco da obesidade, é preciso, portanto, determinar a composição do corpo e definir o tipo de distribuição da gordura corpórea a partir dos seguintes métodos clínicos: antropometria; peso corpóreo; relação peso e estatura; relação cintura quadril e circunferência da cintura ${ }^{(7)}$.

A circunferência abdominal elevada nos portadores de obesidade, em especial, caracteriza-se por uma distribuição abdominal, troncular ou central, estando relacionada com uma série de anormalidades metabólicas, tornando a incidência de DMT2 mais progressiva; geralmente é denominada de Obesidade Andróide. Esta, por sua vez, está relacionada à circunferência da cintura/abdomen, que, também leva a um maior risco de doenças cardiovasculares ${ }^{(3)}$.

A hiperglicemia é uma das principais complicações potenciais do diabetes, e se dá na falta efetiva de insulina, provocando diurese osmótica, com perda de água e eletrólitos. As idades em que mais ocorrem essas alterações são de 50 a 70 anos, e muitas vezes, não há história pessoal ou familiar de diabetes ${ }^{(2)}$.

A história familiar positiva para hipertensão arterial sistêmica é, usualmente, encontrada em pacientes hipertensos, especialmente em pacientes jovens, sendo esta um alerta para o diagnóstico de 
hipertensão arterial sistêmica secundária. Por sua vez, a redução de peso é uma medida anti-hipertensiva, pois a obesidade é um dos maiores fatores de risco para o desenvolvimento de hipertensão, diabetes e eventuais problemas cardíacos $^{(1)}$.

A dislipidemia primária tem origem genética, no entanto, fatores relacionados aos hábitos de vida, como o sedentarismo e a dieta, podem funcionar como fatores desencadeantes para o seu aparecimento. A dislipidemia secundária pode ser originada a partir de doenças como DM, obesidade, hipotireoidismo, insuficiência renal, dentre outras ${ }^{(14)}$.

Os dados evidenciados nesse estudo corroboram com outro estudo ${ }^{(7)}$ que constatou, no geral, o peso de menor risco em adultos com menos de 40 anos corresponde a um IMC entre 20 e $25 \mathrm{~kg} / \mathrm{m}^{2}$ e até 27 para indivíduos mais velhos. O risco com base no IMC está ancorado na tabela de classificação do estado nutricional, que refere: sobrepeso (pré-obeso) com IMC de 25 à $29,9 \mathrm{~kg} / \mathrm{m}^{2}$, Obesidade Grau I (obeso classe I) com IMC de 30 à $34,9 \mathrm{~kg} / \mathrm{m}^{2}$, Obesidade Grau II (obeso classe II) com IMC de 35 à $39,9 \mathrm{~kg} / \mathrm{m}^{2}$ e Obesidade Grau III (obeso classe III) com IMC $\geq 40,0 \mathrm{~kg} / \mathrm{m}^{2}$.

Ainda enfatiza quanto maior o ganho de peso, maior a propensão para aumento de resistência à insulina, menor utilização das células em queimar gordura e açúcar, maior depósito de gordura nos vasos e, portanto, aterosclerose, maior tendência de trombose, diabetes e hipertensão, induzindo à progressiva obliteração dos vasos e degradação da função do coração, podendo levar a morte súbita ${ }^{(7)}$.

\section{CONSIDERAÇÕES FINAIS}

Ao término deste estudo, conclui-se que, nas últimas décadas, o número de pessoas com sobrepeso e obesidade, de forma especial em adultos e idosos, vem crescendo consideravelmente tanto no mundo como no Brasil, sendo esse um grave problema de saúde pública.

Nesta perspectiva, observamos que muitos são os desafios para os profissionais na assistência e acompanhamento dos usuários portadores de obesidade da UBSF, no Tambor I. Todavia, é necessário que os gestores empenhem-se em gerar subsídios que promovam um trabalho integral, com apoio estrutural e didático, fornecendo aos profissionais auxílios para atuar no âmbito preventivo da ESF.

Deste modo, esperamos que este estudo contribua para a assistência prestada pelos profissionais desta
UBSF, com os profissionais de enfermagem e todos aqueles que, de uma forma ou de outra, estejam envolvidos com a assistência aos portadores de obesidade. Sendo assim, constatamos a importância da sensibilização e conscientização destes profissionais, para que se efetive a busca ativa de DM em suas áreas de atuação.

Recomendamos medidas e ações que proporcionem perda de peso dos usuários e ações educativas que abordem essa temática, na tentativa de prevenir os riscos, bem como a adoção de estilos de vida saudáveis (alimentação balanceada e exercícios físicos). Mas, também, aconselhamos a realização de estudos com amostras ainda maiores, para que assim, mais casos sejam detectados.

\section{REFERÊNCIAS}

1. Duncan BB, Schmidt MI, Giuglian ERJ. Medicina ambulatorial: condutas de atenção primária baseadas em evidências. $3^{\mathrm{a}}$ ed. Porto Alegre: Artmed; 2006.

2. Smeltzer SC, Bare BG. Tratado de enfermagem médico-cirúrgico. 9 $9^{\mathrm{a}}$ ed. Rio de Janeiro: Guanabara Koogan; 2002.

3. Sociedade Brasileira de Diabetes, organizadores. Tratamento e acompanhamento do diabetes mellitus. Rio de Janeiro: Diagraph; 2007.

4. Dovera TMDDS. Nutrição aplicada ao curso de enfermagem. Rio de Janeiro: Guanabara Koogan; 2007.

5. Ministério da Saúde (BR). Programas de Agentes Comunitários de Saúde. Brasília (DF): Ministério da Sauúde; 2004.

6. Ministério da Saúde (BR). Departamento de Ações Programáticas Estratégicas. Plano de reorganização da atenção a hipertensão arterial e ao diabetes mellitus: hipertensão arterial e diabetes mellitus. Brasília (DF): Ministério da Saúde; 2001.

7. Cuppari L (Coordenadora). Guia de nutrição: nutrição clínica no adulto. $2^{\mathrm{a}}$ ed. Barueri: Manole; 2007.

8. Gonçalves HA. Metodologia da pesquisa. Campinas: Avercamp; 2005.

9. Ministério da Saúde (BR). Conselho Nacional de Saúde. Diretrizes e normas regulamentadoras de pesquisa envolvendo seres humanos. Resolução n. 
196, de 10 de outubro de 1996. Brasília; 1996.

10. Ministério da Saúde (BR). Obesidade Secretaria de Atenção à Saúde. Departamento de Atenção Básica. Brasília: Ministério da Saúde; 2007.

11. Ministério da Saúde (BR). Secretaria de Atenção à Saúde. Departamento de Atenção Básica. Guia alimentar para a população brasileira: promovendo a alimentação saudável. Brasília: Ministério da Saúde; 2008.

12. Rouquayrol MZF. Epidemiologia e saúde. $6^{\mathrm{a}}$ ed. Rio de Janeiro: MEDSI; 2003.

13. Katch FI, Mcardle WD. Nutrição, controle de peso e exercício. $3^{\mathrm{a}}$ ed. Rio de Janeiro: MEDSI; 1990.

14. Figueiredo DM. Dislipidemiasxsaúde. 2007. [acesso em 20 ago 2009]. Disponível: http://www.portaldiabetes. com.br/conteudocompleto.asp?idconteudo $=486$ 